\title{
Parallel theoretical study of the two components of the prompt fission neutrons: Dynamically released at scission and evaporated from fully accelerated fragments
}

\author{
Nicolae Carjan ${ }^{1,2, a}$, Margarit Rizea ${ }^{2}$, and Patrick Talou ${ }^{3}$ \\ 1 Joint Institute for Nuclear Research, Dubna, Moscow Region, Russia \\ 2 National Institute of Physics and Nuclear Engineering, "Horia Hulubei", Bucharest, Romania \\ 3 T-2 Nuclear Theory Group, LANL, Los Alamos, New Mexico 87544, USA
}

\begin{abstract}
Prompt fission neutrons (PFN) angular and energy distributions for the reaction ${ }^{235} \mathrm{U}\left(n_{t h}, f\right)$ are calculated as a function of the mass asymmetry of the fission fragments using two extreme assumptions:

1) PFN are released during the neck rupture due to the diabatic coupling between the neutron degree of freedom and the rapidly changing neutron-nucleus potential. These unbound neutrons are faster than the separation of the nascent fragments and most of them leave the fissioning system in few $10^{-21}$ sec. i.e., at the begining of the acceleration phase. Surrounding the fissioning nucleus by a sphere one can calculate the radial component of the neutron current density. Its time integral gives the angular distribution with respect to the fission axis. The average energy of each emitted neutron is also calculated using the unbound part of each neutron wave packet. The distribution of these average energies gives the general trends of the PFN spectrum: the slope, the range and the average value.

2) PFN are evaporated from fully accelerated, fully equilibrated fission fragments. To follow the de-excitation of these fragments via neutron and $\gamma$-ray sequential emissions, a Monte Carlo sampling of the initial conditions and a Hauser-Feshbach statistical approach is used. Recording at each step the emission probability, the energy and the angle of each evaporated neutron one can construct the PFN energy and the PFN angular distribution in the laboratory system.
\end{abstract}

The predictions of these two methods are finally compared with recent experimental results obtained for a given fragment mass ratio.

\section{How was the evaporation hypothesis born}

The main properties of the fission neutrons (their multiplicity and their angular and energy distributions) have been initially measured in the frame of the Manhattan project $[1,2]$ and then remeasured in the 50's [3,4]. These properties are:

1) an almost Maxwellian distribution of the fission neutron energies and

2) an angular distribution with respect to the light fragment direction asymmetricaly peaked at $0^{\circ}$ and $180^{\circ}$ (i.e., $\left.v\left(0^{\circ}\right) / v\left(180^{\circ}\right)>1\right)$.

The simplest explanation of these two features was immediately embraced: the fission neutrons are evaporated from moving fission fragments (this was the terminology at that time). The fact that Weisskopf's nuclear evaporation theory [5] existed certainly helped. Strange enough nobody has revendicated this explanation. Nowadays it is reffered as "Los Alamos" or "Madland-Nix" model although this work [6] was published 30 years later.

a e-mail: carjan@theory.nipne.ro
In the wake of this revelation the neutron spectra were fitted with Maxwellian and Watt distributions and, as a bonus, information about the nuclear temperature of the fission fragments was extracted. The asymmetry of the angular distribution with respect to $90^{\circ}$ could not be simply explained by unequal fragment velocities $\left(v_{L}>v_{H}\right)$. An extra parameter was added and fitted to the experimental curve. As a result it was concluded that the light fragment must emit, on the average, about $30 \%$ more neutrons than the heavy one.

\section{An alternative explanation of the main properties of the promptfission neutrons}

It was recently shown [8] that the calculated properties of the neutrons released during a non-adiabatic transition at scission for ${ }^{236} \mathrm{U}$ are in agreement with data on prompt fission neutrons in the reaction ${ }^{235} \mathrm{U}\left(n_{t h}, f\right)$. The calculations were performed in the frame of a dynamical scission model [9] that will be shortly presented in the next section.

To see the quality of the agreement, the calculated angular distribution, with respect to the light fragment direction, of the scission neutrons $(\mathrm{SN})$ is represented 


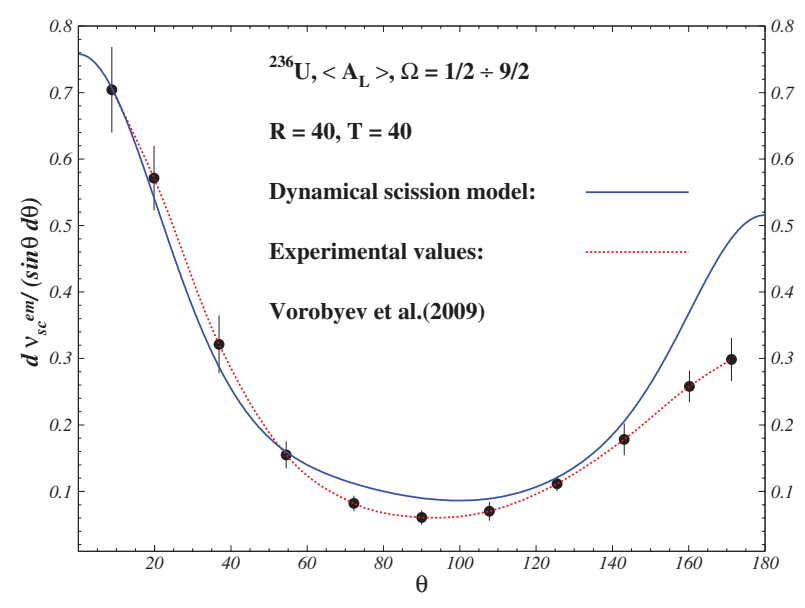

Figure 1. Comparison between the calculated angular distribution with respect to the fission axis of the $\mathrm{SN}$ for ${ }^{236} \mathrm{U}$ and the one measured for all PFN in the reaction ${ }^{235} \mathrm{U}\left(n_{t h}, f\right)$ [10]. The theoretical curve represents an average over all mass ratios.

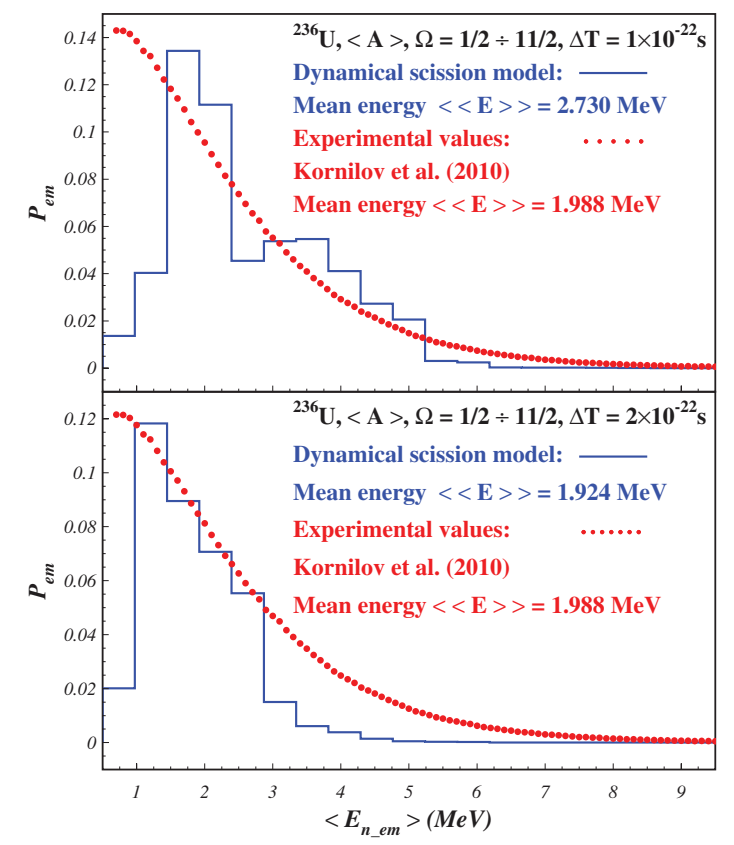

Figure 2. Comparison between the histogram of the $\mathrm{SN}$ average energies calculated for ${ }^{236} \mathrm{U}$ and the energy spectrum measured for all $\mathrm{PFN}$ in the reaction ${ }^{235} \mathrm{U}\left(n_{t h}, f\right)$ [12]. Calculations are performed for two transition times $\Delta T=1$ (above) and 2 (below) $\times 10^{-22} \mathrm{sec}$.

in Fig. 1 together with the distribution measured for all prompt fission neutrons (PFN) [10]. Except a small deviation in the heavy fragment direction, the curves almost coincide. More neutrons are naturally predicted in the direction of the light fragment. A ratio $v_{L}^{e m} / v_{H}^{e m}$ in agreement with the experimental value (1.41) [11] is obtained at $T=40 \times 10^{-22} \mathrm{sec}$.

For the moment we cannot estimate the energy spectrum but we can calculate the average kinetic energy of the scission neutrons emitted from each initial state. Their distribution (represented as a histogram) gives the correct range, slope and average energy of the measured spectrum [12] as seen in Fig. 2.

Concerning the total average multiplicity, $\left\langle v_{p}\right\rangle$, it is easy to reproduce it since this quantity is very sensitive to the pre- and post-scission shapes and to a less extent also to the duration $\Delta \mathrm{T}$ of the scission process [8].

One can say that the agreement is not only surprising but it is also better than expected from a model that uses little-known scission shapes and scission times. If this alternative explanation had existed in the 50's, the field of fission neutrons would have probably known a different development.

\section{The dynamical scission model (DSM)}

The coupling between the neutron degree of freedom and the changing neutron-nucleus potential during the scission process (i.e., from the neck rupture at finite radius $r_{\text {min }}$ to the absorption of the neck stubs by the fragments) was investigated using the time-dependent Schrödinger equation with time-dependent potential [9].

The scission process is mainly defined by the nuclear shapes just before $\left(\alpha_{i}\right)$ and immediatelly after scission $\left(\alpha_{f}\right)$ and by the duration $\Delta T$ of the transition between these two shapes. These quantities are not really known; one can only make educated guesses about them. The lower limit of $\Delta T$ should be about $5 \times 10^{-23} \mathrm{sec}$, the time required for a Fermi level nucleon to cross a $4 \mathrm{fm}$ thick neck. Here we take $\Delta T=1 \times 10^{-22}$ and $2 \times$ $10^{-22} \mathrm{sec}$ that we consider realistic values. The minimum neck radius $r_{\min }$ in the initial configuration predicted by the optimal scission shapes [13] is $\approx 2 \mathrm{fm}$. This is also the value deduced by general considerations like the size of the alpha particle. Here we take a slightly lower value $(1.6 \mathrm{fm})$. There is no indication about the minimum distance between the surfaces of the two fragments $d_{\text {min }}$ in the final configuration. Here we take $0.6 \mathrm{fm}$. When more reliable values for these quantities are available we will use them. For the moment we do not think that it makes sense to adjust them to the data.

Let us consider the neutron wave functions after scission (i.e. at $t=\Delta T) \hat{\Psi}^{i}(\Delta T)$, that correspond at $t=0$ to the eigenstates $\hat{\Psi}^{i}$ that are occupied in the initial configuration $\alpha_{i}$. Their distribution over the eigenstates of the $\alpha_{f}$ configuration is given by

$$
a_{i f}=\left\langle\hat{\Psi}^{i}(\Delta T) \mid \hat{\Psi}^{f}\right\rangle .
$$

$f^{i}=\left|\hat{\Psi}_{e m}^{i}(t)\right\rangle$, the emitted part of $\left|\hat{\Psi}^{i}(t)\right\rangle$, is given by the contribution of the unbound states to the wave packet:

$$
\left|\hat{\Psi}_{e m}^{i}(t)\right\rangle=\left|\hat{\Psi}^{i}(t)\right\rangle-\sum_{\text {bound states }} a_{i f}\left|\hat{\Psi}^{f}\right\rangle
$$

The corresponding current density weighted by the occupation probability $v_{i}^{2}$ of the respective state $\mathrm{i}$ :

$$
\bar{D}_{e m}(\rho, z)=\frac{i \hbar}{\mu} \sum_{i} v_{i}^{2}\left(f^{i} \bar{\nabla} f^{i *}-f^{i *} \bar{\nabla} f^{i}\right),
$$

provides the distribution of the average directions of motion of the unbound neutrons at any time $t$.

To calculate the angular distribution of the SN with respect to the fission axis one needs to integrate in time the radial component of $\bar{D}_{e m}$ along the surface of a sphere of radius $R$ containing the fissioning nucleus:

$$
d \nu_{s c}^{e m} /(\sin \theta d \theta)=4 \pi \int_{0}^{T} \bar{D}_{e m}(R, \theta, t) \bar{n}(R, \theta) R^{2} d t
$$


$\bar{n}$ is the unit vector perpendicular to the surface. For $R$ we choose $30 \mathrm{fm}$ and $40 \mathrm{fm}$. In the calculations with $R=40 \mathrm{fm}$ we also improved the Woods-Saxon potential at scission by replacing the gradient approximation [14] with an exact calculation of the distance to the nuclear surface [15]. The upper limit of the time integral should be in principle $\infty$. In practice we can reach only a finite value $T=4 \times$ $10^{-21} \mathrm{sec}$.

The scission neutron multiplicity $v_{s c}$ is given by the sum of the probabilities $P_{e m}^{i}$ that a neutron occupying a given bound-state $i$ is released immediately after scission i.e. $t=\Delta T$ :

$$
v_{s c}=2 \sum_{i} P_{e m}^{i}, P_{e m}^{i}=v_{i}^{2}\left(\sum_{f}\left|a_{i f}\right|^{2}\right)
$$

The i-sum is over bound states while the f-sum is over unbound states.

The average kinetic energy of each emitted neutron is:

$$
E_{n}^{i}=\left\langle\hat{\Psi}_{e m}^{i}(\Delta T)\left|\hat{\mathcal{H}}\left(\alpha_{f}\right)\right| \hat{\Psi}_{e m}^{i}(\Delta T)\right\rangle .
$$

$\hat{\mathcal{H}}$ is the single-particle Hamiltonian with extremely deformed Woods-Saxon mean field and related spin-orbit potential used at $\alpha_{f}$.

The function $P_{e m}^{i}\left(E_{n}^{i}\right)$ gives the distribution of the $\mathrm{SN}$ average energies.

\section{CGMF: An advanced evaporation code}

The CGMF code [16] is a Monte Carlo implementation of the well-known Hauser-Feshbach statistical theory of nuclear reactions, applied to the de-excitation of the fission fragments. In its current version, CGMF assumes that there is no pre-scission neutron emission, except at higher energies in the multi-chance fission process, and that all neutron and $\gamma$-ray evaporations happen only after the two complementary fragments reach full acceleration. The mean excitation energy of the fission fragments in lowenergy fission reactions is typically 15 to $20 \mathrm{MeV}$. At those excitation energies, only neutron and $\gamma$ evaporations are allowed, light-charged particle emissions being hindered by the Coulomb barrier. The fragments will shed most of their excitation energy through neutron evaporation, followed by $\gamma$-ray decays until a ground or isomeric state is reached.

As for any other Hauser-Feshbach nuclear reaction code, the decay of an excited compound nucleus, here a fission fragment, is governed by emission probabilities. The emission probability of a neutron of energy $\epsilon_{n}$ from an initial nucleus $(Z, A)$ characterized by an energy $U_{i}$, spin $J_{i}$, and parity $\pi_{i}$ is given by

$$
P_{n}\left(\epsilon_{n}\right) d \epsilon_{n} \propto T_{n}\left(\epsilon_{n}, l, j\right) \rho\left(A-1, Z, U_{f}, J_{f}, \pi_{f}\right) d \epsilon_{n},(6)
$$

the final energy in the residual nucleus given by $U_{f}=U_{i}-\epsilon_{n}-B_{n}$, final spin $J_{f}$ and final parity $\pi_{f}$. The neutron transmission coefficients $T_{n}\left(\epsilon_{n}, l, j\right)$ are obtained as usual from optical model calculations of the neutron+residual nucleus interaction. Spin and parity selection rules are applied at each stage of the decay. The level density $\rho$ entering in Eq. (6) is a short-cut notation to describe a set of discrete excited states at low

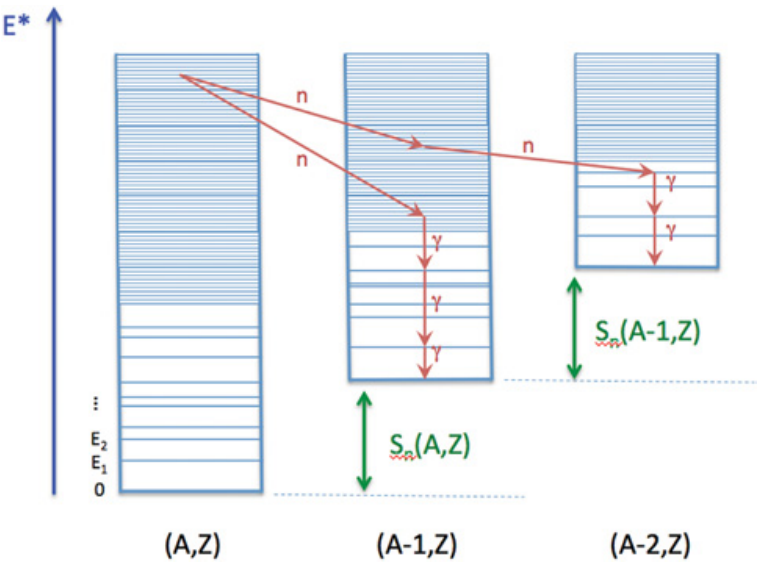

Figure 3. Schematic representation of two different decay paths of a particular nucleus, sampled according to computed emission probabilities for neutrons and $\gamma$ rays.

energies, followed by a continuum representation at higher energies, where individual states cannot be distinguished anymore. Known nuclear levels for the fission fragments are taken from the RIPL-3 database [17]. Nuclear level density parameter systematics are also taken from RIPL-3.

Prompt $\gamma$-ray emission probabilities are obtained similarly as

$$
P_{\gamma}\left(\epsilon_{\gamma}\right) d \epsilon_{\gamma} \propto T_{\gamma}\left(\epsilon_{\gamma}\right) \rho\left(A, Z, U_{f}, J_{f}, \pi_{f}\right) d \epsilon_{\gamma},
$$

with $U_{f}=U_{i}-\epsilon_{\gamma}$. The $\gamma$-ray transmission coefficients $T_{\gamma}\left(\epsilon_{\gamma}\right)$ are obtained in the strength function formalism for the $E 1, E 2$ and $M 1$ multipolarities, following the Kopecky-Uhl representation.

As mentioned earlier, CGMF uses the Monte Carlo technique to perform this calculation. At each stage of the decay, characterized by a specific nucleus in a specific energy, spin and parity configuration, CGMF calculates the neutron and $\gamma$ emission probabilities, and then sample those. In this way, different decay paths are generated at each new CGMF run, as shown schematically in Fig. 3. By performing a large number of CGMF computations, "histories" of fission events can be recorded, similarly to what is done with list-mode data acquisition in modern experiments.

The outcome of any evaporation model depends on the partition of the total excitation energy among the light and the heavy fragment which is unknown. It is usually parametrized by the ratio $T_{L} / T_{H}$ of the fragments' temperatures. In the present calculations we use the assumption of thermal equilibrium [6].

\section{Comparison with experimental data for fixed fragment masses}

In all models the PFN spectra and angular distributions are calculated separately for each fragment-mass ratio. On the other hand, the experimental distributions are either inclusive or, if selected for a given mass ratio, do not have enough statistics. So far the comparison has been therefore possible only for results integrated over all fragment masses. Integrated distributions are usually smooth and do not allow the distinction between different model assumptions. 


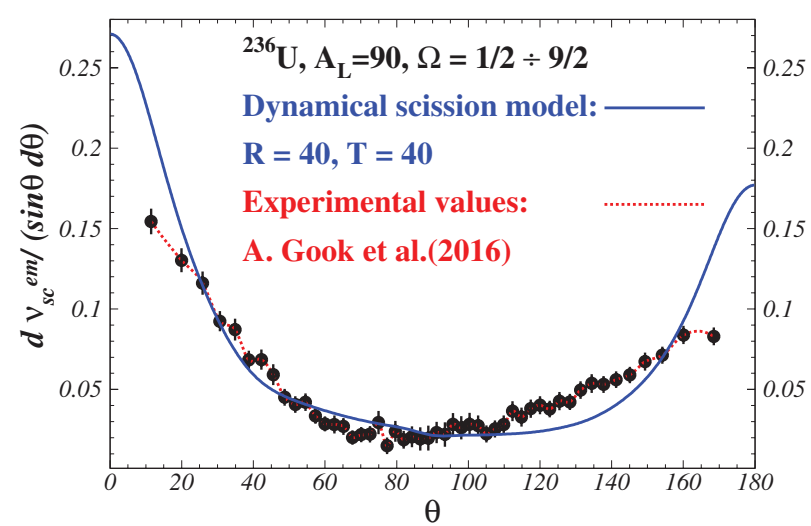

Figure 4. Angular distribution with respect to the light-fragment direction calculated with DSM for the fragment-mass ratio $A_{L} / A_{H}=90 / 146$.

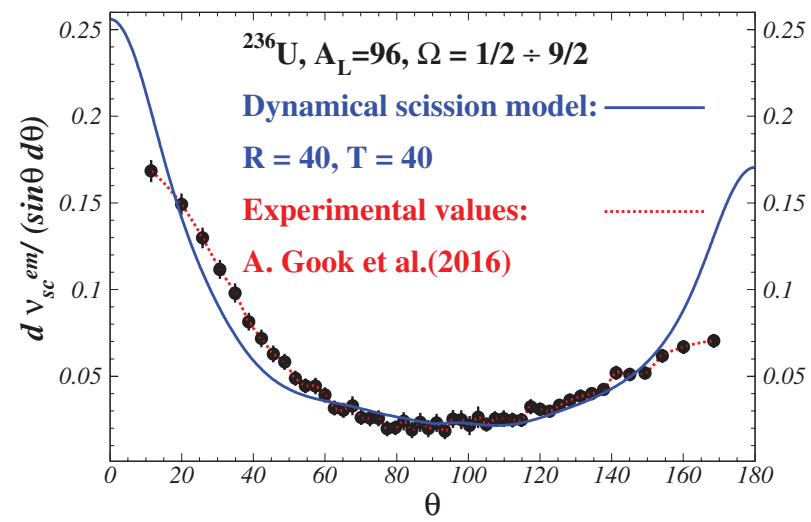

Figure 5. Angular distribution with respect to the light-fragment direction calculated with DSM for the fragment-mass ratio $A_{L} / A_{H}=96 / 140$.

In a recent experiment [18], with improved angular (FWHM $=7 \mathrm{deg}$ ) and mass resolution ( $3 \mathrm{amu})$, enough events have been accumulated to construct distributions for each mass ratio. A comparison with such data represents obviously a more powerful test of the PFN emission mechanism.

\subsection{Neutrons dynamically emitted at scission}

Equation (3) is used to calculate the angular distribution for fixed mass ratios defined by the mass of the light fragment $A_{L}$. It is then convoluted with a Gaussian resolution function. The results for $A_{L}=90$ and $A_{L}=96$ are compared in Figs. 4 and 5 respectively with recent measurements [18]. These data are preliminary in the sense that only $1 / 3$ of the events collected have been analyzed so far.

Two features are however worth mentioning:

a) In the range (40-140) deg the data reveal structures that could be due to scission-neutron scattering on nascent fragments.

b) In the direction of the fragments (i.e., in the ranges (0-40) deg and (140-180) deg the distribution is rather flat. It is flatter on the heavy fragment side. This could be a sign of scission-neutron reabsorption by the fission fragments with the heavy fragment having a stronger effect.

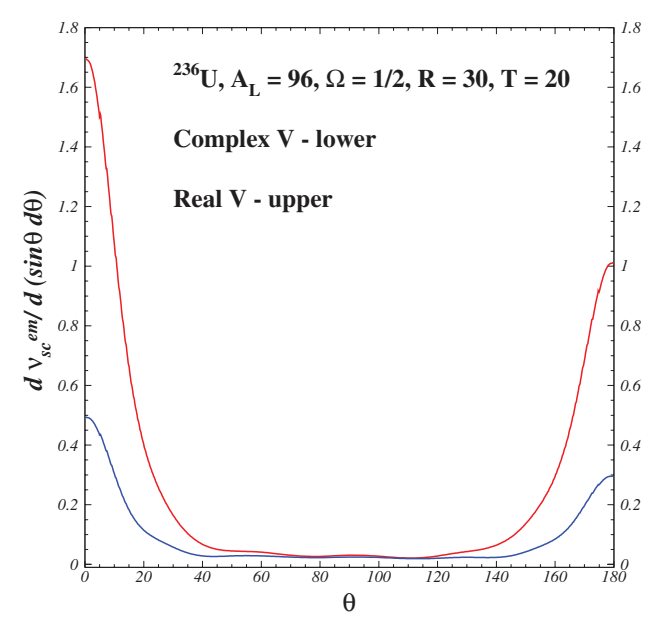

Figure 6. Effect of the imaginary potential $(W=2 \mathrm{MeV})$ on the angular distribution calculated with DSM.

Figure 6 shows the effect of the imaginary potential on the SN angular distribution. As expected it reduces the scission neutron flux in the direction of the fragments while the neutrons moving perpendicular to the fission axes are unaffected. In this particular example, the oscillations between 0 and 40 deg are very small. There are a number of reasons for this:

a) Only states with $\Omega=1 / 2$ (the projection of the angular momentum on the nuclear axes) are taken into account. These states represent the majority but contribute predominantly to angles around 0 and $180 \mathrm{deg}$. Neutrons with higher $\Omega$ values are emitted at intermediate angles and are responsible for the oscillations.

b) The upper limit of the time integral in Eq. (3) is relatively short: $T=20 \times 10^{-22} \mathrm{sec}$. As shown in Ref. [19] the oscillations are slightly increasing in time.

c) In the DSM the initial neutron source is partly located inside the fragments and therefore not all neutrons released during scission are able to scatter. Using a pedagogical model with simplified initial conditions $[20,21]$, the case of two well-separated spherical fragments with a neutron source inbetween was studied. The amplitude of the oscillations at intermediate angles turned out to be comparable with the yields in the direction of the fission fragments.

Equation (5) is used to calculate the average kinetic energy of a scission neutron emitted from a given state $i$. Knowing also the probability $P_{e m}^{i}$ that such a neutron is released, we can obtain the distribution of the $\mathrm{SN}$ average energies. The results, for the same mass ratios as before, are presented in Figs. 7 and 8 together with recently measured prompt fission-neutron spectra. Since, at the present stage, we have not calculated for each state $i$ the full spectrum but only its average value, the comparison between theory and experiment can be made only for the gross properties: the range, the average value and the slope.

For the full spectrum we would need to calculate the Fourier transform (FT) of each emitted wave function $\left|\hat{\Psi}_{e m}^{i}(\Delta T)\right\rangle$, to add the contributions from all occupied states and finally obtain the momentum $(K)$ 


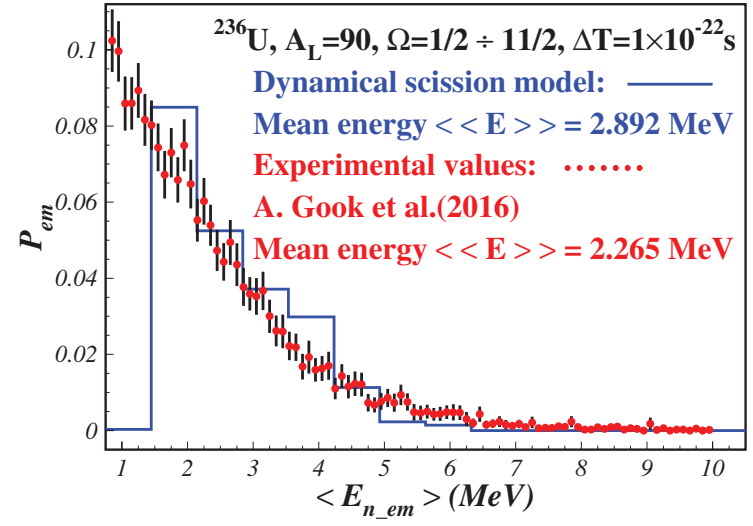

Figure 7. Histogram of scission neutron average energies calculated with DSM for the fragment-mass ratio $A_{L} / A_{H}=$ 90/146.

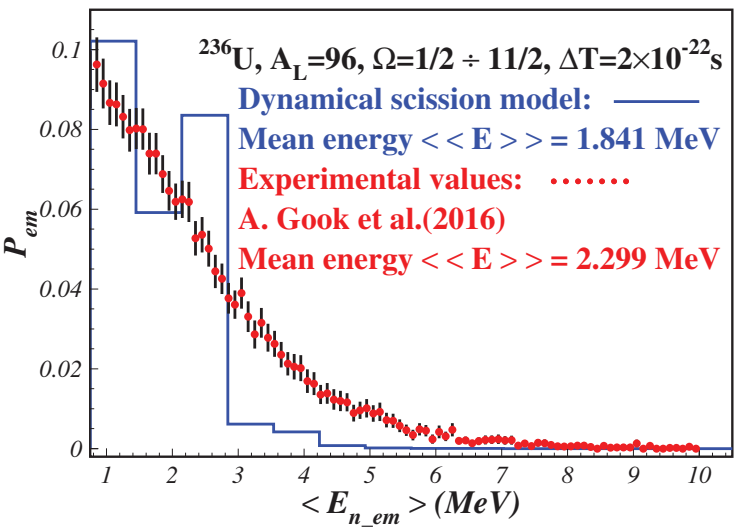

Figure 8. Histogram of scission neutron average energies calculated with DSM for the fragment-mass ratio $A_{L} / A_{H}=$ 96/140.

distribution. The formalism and the numerical procedure to calculate the FT's of neutron wave functions in extremely deformed nuclei (that are usually expressed in cylindrical coordinates) became only very recently available [22].

The emitted part of the wave function is a sum of discrete states in the continuum which have several radial nodes. The corresponding FT's will lead to a $K$-distribution with several oscillations which will continue to exist even after summing the spectra of all states. Therefore the full SN spectrum for a given mass ratio is expected to exhibit small oscillations superposed on a qausi-exponential decrease.

If the structures observed in the above mentioned data are not washed out when more statistics is added we will have a valuable indication regarding the origin of PFN.

\subsection{Neutrons evaporated from fully accelerated fragments}

The Hauser-Feshbach Monte-Carlo technique described in Sect. 4 is applied to the de-excitation of each of the two fragments by isotropic emission of neutrons and $\gamma$-rays. The two fragments are supposed to have the same temperature. A transformation from the CM system of each fragment to the LAB system is finally performed.

The corresponding angular distributions of the evaporated neutrons for four mass divisions chosen
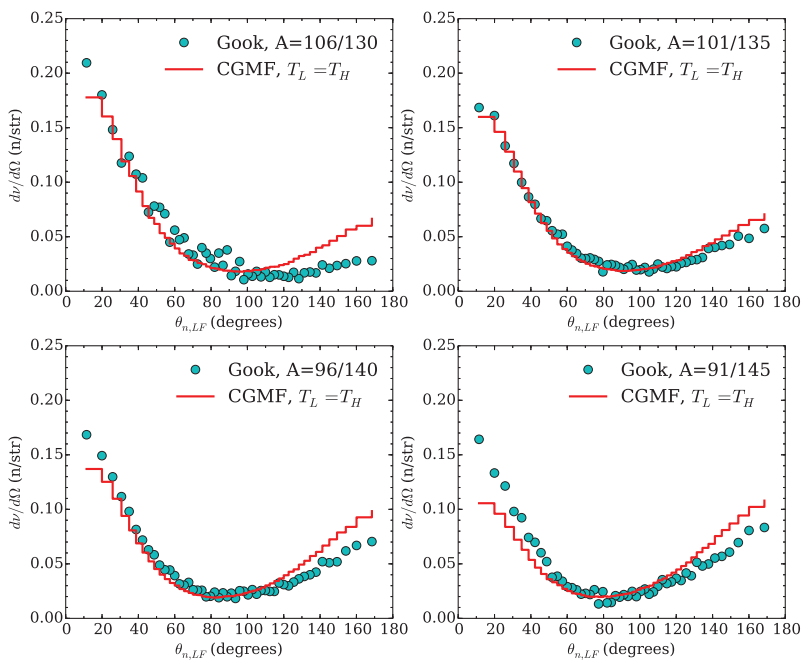

Figure 9. Angular distribution with respect to the light-fragment direction calculated with CGMF for selected fragment-mass ratios.
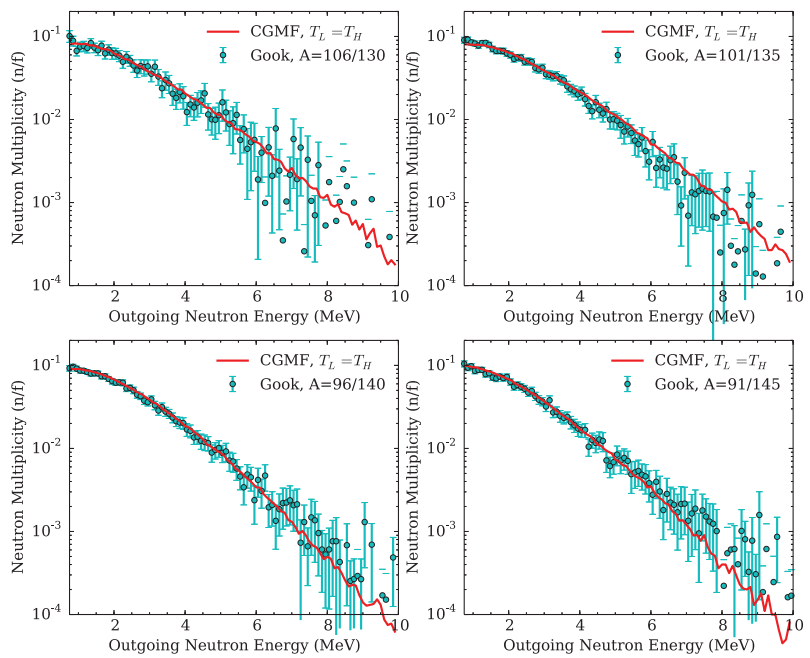

Figure 10. Energy distribution in the laboratory system calculated with CGMF for selected fragment-mass ratios.

around the most probable one $\left(A_{L} / A_{H}=96 / 140\right)$ are presented in Fig. 9. It is known that the hypotheses of thermal equilibrium at scission doesn't reproduce the measured ratio $v_{p}^{L} / v_{p}^{H}=1.4$. On the contrary it predicts more neutrons to be evaporated by the heavy fragment. Deviations are therefore expected in the direction of the heavy fragment. The only way to obtain agreement between the evaporation hypotheses and the experimental data is by fitting the ratio $T_{L} / T_{H}$ to the measured saw-tooth curve $v_{p}\left(A_{F}\right)$.

The corresponding kinetic energy spectra of evaporated neutrons for the same mass divisions are presented in Fig. 10 on a logarithmic scale. One can see that for mass ratios 96/140 and 91/145 the calculated spectrum is too soft. For more symmetric divisions the errors at high energies are too large to draw any conclusion. In order to analyze better the low energy part, the same results are shown in Fig. 11 on a linear scale. For the mass ratio 101/135 the calculations slightly underestimate the data but otherwise the overall agreement is very good. 

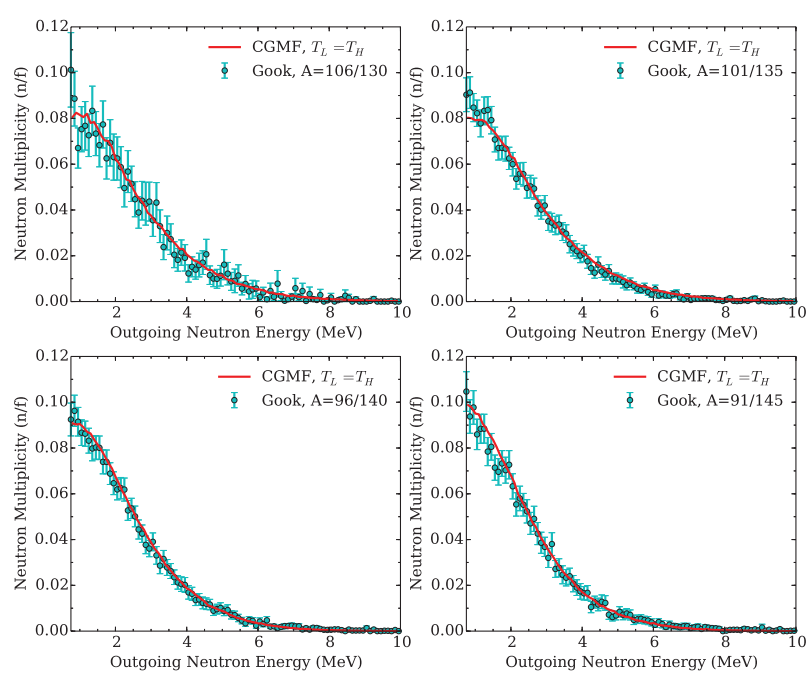

Figure 11. Energy distribution in the laboratory system calculated with CGMF for selected fragment-mass ratios.

\section{Discussions and conclusions}

Although opposed, both models reproduce the gross properties of the main PFN observables that were known in the 50's and on the basis of which the evaporation hypothesis was founded. In the details, CGMF, with adjusted parameters, does a better job.

There is nothing wrong with the evaporation hypotheses except that it is put first. When trying to understand the origin of PFN one should think in chronological order. First comes scission. If this process is diabatic, there is no doubt that neutrons will be released (as are protons and $\alpha$ particles). The amount of scission neutrons depends on the speed and on the length of the scission jump. Some will be reabsorbed when they cross the fragments but the rest will leave the fissioning system during the acceleration phase.

DSM calculations shows [9] that the emission takes time: after $4 \times 10^{-21} \mathrm{sec}$ only $65 \%$ of the released neutrons left the sphere of radius $\mathrm{R}=40 \mathrm{fm}$. However by the time the fragments reach $90 \%$ of their TKE (i.e., $10^{-20} \mathrm{sec}$ ) almost all scission neutrons left that sphere. Now the fragments are well apart, somewhat excited and fully equilibrated; they will evaporate some neutrons and $\gamma$ rays.

The condition that all PFN are evaporated (as commonly believed) is an adiabatic (slow) scission process that leaves the primary fragments well deformed. The corresponding transition time was estimated to be $5 \times$ $10^{-22}$ [23] i.e., one order of magnitude larger than the shortest possible transition time. There is a priori no reason to exclude such a long time but then we should figure out why scission protons and $\alpha$ 's are emitted.

It is also possible that the large majority of PFN are released at scission. For this to happen we need the scission process: (a) to be diabatic (as in the present study) and (b) to leave the primary fragments close to their groundstate deformation (longer jump than in the present study). Even in this case the primary fragments are excited due to the same diabatic coupling that is responsible for releasing scission neutrons. As a whole this excitation energy is of the order of $12 \mathrm{MeV}$ [24] but per nucleonic degree of freedom is less than the neutron binding. It can be used only to emit prompt $\gamma$ rays. Some excitation can also be generated through re-absorption of released neutrons by the fragments at the beginning of their acceleration. In principle this extra excitation energy can also be used to emit $\gamma$ 's and eventually to evaporate neutrons.

Clarifying the role of scission neutrons as part of PFN has a win-win outcome. Irrespectiv of the answer we will deduce exclusive information about the last fission stage such as: its degree of adiabaticity and the nuclear configurations involved.

Part of this work was performed at Los Alamos National Laboratory, under the auspices of the National Nuclear Security Administration of the U.S. Department of Energy at LANL under Contract No. DE-AC52-06NA25396.

\section{References}

[1] F. Bloch, H. Staub, U. S. Atomic Energy Commission Document AECD-3158, 1943

[2] R.R. Wilson, 1945, published in Phys. Rev. 77, 189 (1947)

[3] J.S. Fraser, Phys. Rev. 88, 536 (1952)

[4] J. Terrell, Phys. Rev. 113, 527 (1959)

[5] V.F. Weisskopf, Phys. Rev. 52, 295 (1937)

[6] D.G. Madland, J.R. Nix, Nucl. Sci. Eng. 81, 213 (1982)

[7] N. Carjan, P. Talou and O. Serot, Nucl. Phys. A 792, 102 (2007)

[8] N. Carjan, M. Rizea, Phys. Lett. B 747, 178 (2015)

[9] M. Rizea, N. Carjan, Nucl. Phys. A 909, 50 (2013)

[10] A.S. Vorobyev, O.A. Scherbakov et al., Nucl. Instr. Meth. A 589, 795 (2009)

[11] K. Nishio et al., Nucl. Phys. A 632, 540 (1998)

[12] N. Kornilov, F.-J. Hambsch et al., Nucl. Sci. Eng 165, 117 (2010)

[13] F.A. Ivanyuk, K. Pomorski, Phys. Rev. C 79, 054327 (2009)

[14] V. Pashkevich, Nucl. Phys. A 169, 275 (1971)

[15] R. Capote, N. Carjan, S. Chiba, Phys. Rev. C 93, 024609 (2016)

[16] P. Talou, T. Kawano, I. Stetcu, CGMF User Manual, Los Alamos Technical Report, LA-UR-14-24031

[17] R. Capote et al., Nuclear Data Sheets 110, 3107 (2009)

[18] A. Göök, F.-J. Hambsch, W. Geertz, M. Vidali, private communication

[19] M. Rizea, N. Carjan, Proc. Rom. Acad. A 16, 176 (2015)

[20] T. Wada et. al, Phys. Proc. 47, 33 (2013)

[21] T. Wada, T. Asano, N. Carjan, Phys. Proc. 64, 34 (2015)

[22] M. Rizea, N. Carjan, Eur. Phys. J. A to be published

[23] N. Carjan, M. Rizea, Int. J. Mod. Phys. E 21, 1250031 (2012)

[24] N. Carjan, F.-J. Hambsch, M. Rizea, O. Serot, Phys. Rev. C 85, 044601 (2012) 